\title{
RESEARCH
}

\section{Comparison of simulated periodontal bone defect depth measured in digital radiographs in dedicated and non-dedicated software systems}

\author{
G Scaf*, ${ }^{*}$, CE Sakakura ${ }^{1}$, PFD Kalil ${ }^{1}$, JAN Dearo de Morais $^{1}$, LCM Loffredo $^{2}$ and A Wenzel ${ }^{3}$ \\ ${ }^{1}$ Department of Oral Diagnosis and Surgery, Araraquara Dental School, State of São Paulo University, Unesp, Araraquara, São Paulo, \\ Brazil; ${ }^{2}$ Department of Social Dentistry, Araraquara Dental School, State of São Paulo University, Unesp, Araraquara, São Paulo, \\ Brazil; ${ }^{3}$ Department of Oral Radiology, School of Dentistry, Faculty of Health Sciences, University of Aarhus, Denmark
}

\begin{abstract}
Objectives: To compare simulated periodontal bone defect depth measured in digital radiographs with dedicated and non-dedicated software systems and to compare the depth measurements from each program with the measurements in dry mandibles.

Methods: Forty periodontal bone defects were created at the proximal area of the first premolar in dry pig mandibles. Measurements of the defects were performed with a periodontal probe in the dry mandible. Periapical digital radiographs of the defects were recorded using the Schick sensor in a standardized exposure setting. All images were read using a Schick dedicated software system (CDR DICOM for Windows v.3.5), and three commonly available non-dedicated software systems (Vix Win 2000 v.1.2; Adobe Photoshop 7.0 and Image Tool 3.0). The defects were measured three times in each image and a consensus was reached among three examiners using the four software systems. The difference between the radiographic measurements was analysed using analysis of variance (ANOVA) and by comparing the measurements from each software system with the dry mandibles measurements using Student's $t$-test.

Results: The mean values of the bone defects measured in the radiographs were $5.07 \mathrm{~mm}$, $5.06 \mathrm{~mm}, 5.01 \mathrm{~mm}$ and $5.11 \mathrm{~mm}$ for CDR Digital Image and Communication in Medicine (DICOM) for Windows, Vix Win, Adobe Photoshop, and Image Tool, respectively, and $6.67 \mathrm{~mm}$ for the dry mandible. The means of the measurements performed in the four software systems were not significantly different, ANOVA $(P=0.958)$. A significant underestimation of defect depth was obtained when we compared the mean depths from each software system with the dry mandible measurements $(t$-test; $P \cong 0.000)$.

Conclusions: The periodontal bone defect measurements in dedicated and in three non-dedicated software systems were not significantly different, but they all underestimated the measurements when compared with the measurements obtained in the dry mandibles.
\end{abstract}

Dentomaxillofacial Radiology (2006) 35, 422-425. doi: 10.1259/dmfr/61300663

Keywords: digital radiography; alveolar bone loss, measurement

\section{Introduction}

The use of digital radiography is increasing and several systems based on either solid state sensors or photostimulable phosphor plates are available for dentistry with a substantial scientific validation. ${ }^{1-4}$ Despite the advantages offered by these technologies, such as the elimination of chemical processing, lower radiation doses to the patient,

*Correspondence to: Dr. Gulnara Scaf, Rua Humaitá, 1680, 14801-903, Araraquara, São Paulo, Brazil; E-mail: scaf@foar.unesp.br

Received 8 August 2005; revised 19 December 2005; accepted 28 December 2005 image processing and improved image management, the possibility of easy and fast transmission of image data to third parties over the internet could be considered one of the main advantages, offering easy access for the dentist to second opinions on diagnosis and treatment planning. ${ }^{5}$

The availability of several digital dental radiography systems (www.odont.au.dk/rad/Digitalx.htm) has introduced new problems concerning image exchange since there is no absolute standard in dentistry for image file format. This means that digital images acquired in one 
system are not easily imported into another system's software. Efforts are ongoing to implement the DICOM (Digital Image and Communication in Medicine) standard in dentistry to encourage interchangeability among software systems. ${ }^{6}$ However, few dental digital systems have offered a DICOM solution, ${ }^{7}$ which moreover may not be the right solution for dentistry. ${ }^{8}$ Furthermore, only a minor percentage of dental clinics have yet switched from traditional film-based radiography to digital imaging. ${ }^{9,10}$

However, increasing numbers of dentists have a personal computer and internet facilities in their offices and there are several low cost software packages available on the market to import and display digital images. These include freeware, e.g. Image Tool 3.0 available at http://ddsdx. uthscsa.edu/dig/itdesc.html. It may be reasonable to believe that dedicated software that comes with a digital system, responsible for capturing digital radiographic images, would be optimized for image interpretation acquired and displayed with this receptor. However, there is a lack of studies addressing the interoperability among different imaging software systems.

The aims of this study were to compare simulated periodontal bone defect depth measured in digital radiographs in dedicated and non-dedicated software systems and to compare depth measurements in each program with those in dry mandibles.

\section{Materials and methods}

Forty dry pig hemi-mandibles were used in this study. Periodontal two-wall bone defects were created at the mesial side of the first premolar in the apical direction using a slow-speed round bur no. 4. The mesial side was chosen because it is the proximal site that gives the best access to create the periodontal bone defect since it presents a diastema between the two contiguous teeth. The bone defect width was determined by the bur diameter and the defect depth was random. Bone was removed corresponding to the bur diameter, and a small chisel was used to refine the defect eliminating all bone chips attached to the root surface. The buccal wall and part of the proximal wall were removed, thus only the lingual wall remained. A tiny mark was made at the cementoenamel junction with a double-face diamond disc to establish the upper border of the bone defect, and it was used as a reference point because the detection of the radiographic image of the cementoenamel junction was difficult.

\section{Image acquisition}

Digital images of the defects were acquired using the CMOS Schick ${ }^{\circledR}$ sensor (Schick Technologies Inc., Long Island, NY). The geometric alignment between the sensor and the hemi-mandible was standardized using a fixing device. A $9.22 \mathrm{~mm}$ long aluminium step wedge was fixed to the sensor, and was also used to calculate the magnification and distortion in the further analysis using an object with a known length. The radiographs were taken with the vertical long axis of the hemi-mandible fixed perpendicular to the central ray and parallel to the sensor at a $70 \mathrm{~cm}$ focal spot to object distance. The X-ray unit operated at $70 \mathrm{kVp}, 10 \mathrm{~mA}$ and 18 impulses. A wooden block of $2 \mathrm{~cm}$ thickness was placed in front of the mandible to simulate soft tissue.

The images were stored in TIFF (Tagged Image File Format) without compression (8 bits with a resolution of $600 \mathrm{dpi}$, a file of about $700 \mathrm{~KB})$.

\section{Bone defect measurement}

The bone defect depth was measured as the distance from the deepest part of the defect to the cementoenamel junction. A periodontal probe (Williams Probe; Hu-Friedy, Chicago, IL) was used to measure the defects in the dry pig mandibles, positioned by hand at the bottom of the defect with the vertical long axis parallel to the root surface. The cementoenamel junction point could be demarcated on the probe with the aid of a small endodontic rubber ring, and the length between the probe tip and the rubber ring was measured by a digital calliper (Ultra-Call Mark III; Fowler, Switzerland) (Figure 1a). This measurement was considered the true depth (gold standard).

The digital images were evaluated in four image software systems (Figure 1b); one, the dedicated Schick program CDR DICOM for Windows v.3.5, and another three non-dedicated software systems: Vix Win 2000 v.1.2, Image Tool 3.00 and Adobe Photoshop 7.0 (Table 1). The software was installed in a laptop computer (Satellite P25S507; Toshiba, Taiwan), and the images were evaluated on a $17^{\prime \prime}$ WXGA $(1440 \times 900)$ monitor.

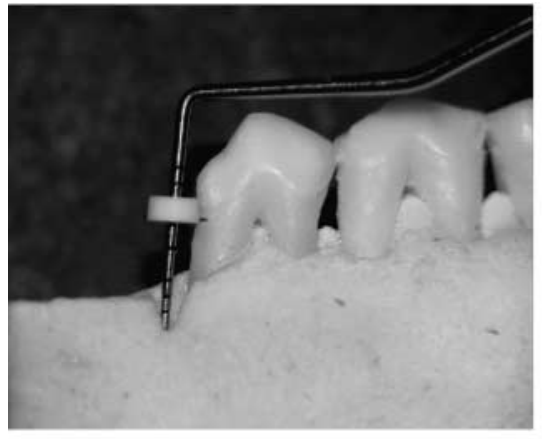

a

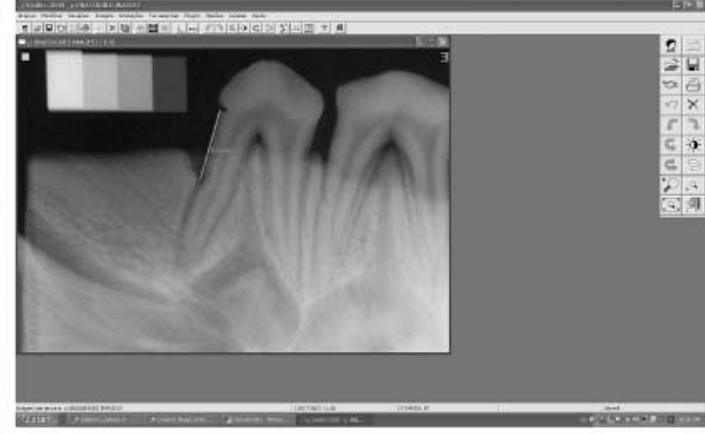

b

Figure 1 Measurement in a dry pig hemi-mandible (a) using periodontal probe and endodontic rubber ring and (b) digital radiographic image showing the distance from the deepest part of the defect to the cementoenamel junction 
Table 1 General information about image software used in this study

\begin{tabular}{|c|c|c|c|c|c|}
\hline Software name & Company & Website & Dedicated & Type & Application \\
\hline Schick DICOM & Schick Technologies & www.schicktech.com & $\mathrm{CDR} \circledast$ & CMOS & Dental \\
\hline Vix Win PRO & Gendex Dental System & www.gendex-dental.com & DenOptix/VisualiX & $\mathrm{PSP} / \mathrm{CCD}$ & Dental \\
\hline Image Tool 3.0 & UTHSCSA & ddsdx.uthscsa.edu/dig/itdesc.html & - & - & Dental/biological \\
\hline Photoshop 7.0 & Adobe & www.adobe.com & - & - & General image processing \\
\hline
\end{tabular}

CCD, charge coupled device; CMOS-sensor, complementary metal oxide semiconductor; PSP, photo stimulable phosphor plate

Prior to the measurements of the defect depths in the digital images, all the images were standardized according to a known length from the step wedge and the ruler tool available in the software system.

The depth measurements were performed by three examiners: one radiologist, one $\mathrm{PhD}$ student and one undergraduate student, for both the dry mandibles and the digital images. The examiners were calibrated before reading sessions and performed the measurements together, in three consecutive sessions, in dimmed room light.

\section{Statistical analysis}

The three measurements of each defect were averaged, and a mean value was obtained. Analysis of variance (ANOVA) was used to compare the depth measurements from the four software systems. Student's $t$-test was used to compare the depth measurements between each method and the dry mandible. The $95 \%$ confidence intervals for the mean depth measurements obtained from each software and dry mandibles were estimated.

STATA software (Stata Corp. 2003. Stata Statistical Software: Release 8.0. College Station, TX: Stata Corporation, TX) was used.

\section{Results}

The ANOVA (Table 2) showed that the means of the measurements performed in the four software systems were not significantly different $(P=0.958)$.

Comparing the radiographic measurements performed in each of the four software systems to the dry mandibles, all digital image measurements underestimated the defect depth ( $t$-test; $P \cong 0.000$ ). The mean measurements were 5.07 (95\% CI: 4.82-5.32), 5.06 (95\% CI: 4.80-5.32), 5.01 (95\% CI: 4.75-5.27), and 5.11 (95\% CI: 4.84-5.37) and 6.67 (95\% CI: 6.30-7.04) for Schick CDR DICOM dedicated software, VixWin, Adobe Photoshop and Image Tool non-dedicated software and dry mandibles, respectively.

The bar graph (Figure 2) shows the difference between the measurements from dry mandibles (gold standard) in each one of the four imaging software systems.

\section{Discussion}

There were two important findings in this study: (1) that measurements of simulated periodontal bone defects obtained in one dedicated and three non-dedicated software systems were not significantly different; (2) the bone defect depth measurements in the digital radiographs were underestimated compared with the measurements obtained with the dry mandibles.

It is important to consider the nature and the facilities provided in software systems developed for dental tasks (Schick, VixWin and Image Tool) and for general applications (Adobe Photoshop). For the latter, there is no specific tool for image standardization, and the standardization in the present study was made using a known length of the aluminium step wedge. On the other hand, Schick, VixWin and Image Tool software systems provide a specific tool for standardization, which is automatically prompted by the system in VixWin and Image Tool when the image is opened on the monitor. In the Schick system, it is necessary for the user to search for this function. Thus, considering distance measurements, the professional can choose each of these software systems, both for dental tasks and for general applications.

For radiographic image interpretation using dedicated and non-dedicated software, the results may be dependent on the diagnostic tasks.

It seems that few studies have evaluated the interoperability among software systems. One study assessed the diagnostic accuracy of the morphology and position of mandibular third molars in digital panoramic images using the dedicated software and a general Picture Archiving and Communication System (PACS) software. The results were similar for the dedicated and the non-dedicated software for all diagnostic tasks, except for the interpretation of impacted third molars, for which the dedicated software gave more accurate results. ${ }^{11}$ However, it is difficult to compare our results with this study, since third molar assessment is a different task from periodontal bone defect measurements.

The results showed that digital radiographic measurements using either of four software systems significantly underestimated the defect depth measured in the dry

Table 2 One-way analysis of variance for depth measurements data according to different software systems

\begin{tabular}{lrccc}
\hline Source of variation & $d f$ & Sum of squares & Mean square & P-value \\
\hline Programs & 3 & 0.2039 & 0.0679 \\
Residual & 156 & 102.6754 & 0.6582 \\
Total & 159 & 102.8793 & & 0.10 \\
\hline
\end{tabular}

df, degrees of freedom; ns, non-significant 


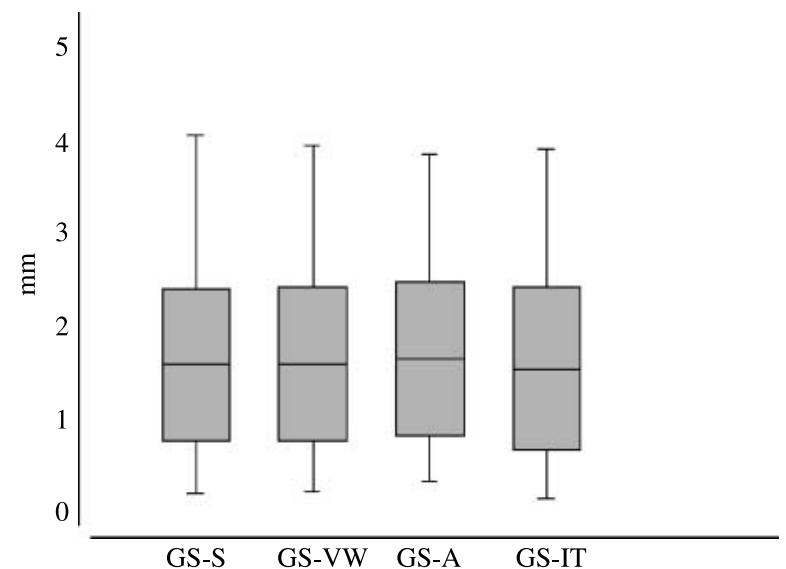

Figure 2 Bar graph of the differences between the gold standard measurements and radiographic measurements in four image softwares. GS, gold standard; S, CDR DICOM v.3.5; VW, Vix Win 2000 v.1.2; A, Adobe Photoshop 7.0; IT, Image Tool 3.0

mandible. The depth measured in radiographs was approximately $25 \%$ lower than the dry mandible measurements. Previous reports have demonstrated an underestimation ranging between $5.7 \%$ and $21 \%$ in bone defects. $^{12-14}$ The explanation for such underestimation

\section{References}

1. Hintze $H$, Wenzel A. Influence of the validation method on diagnostic accuracy for caries. A comparison of six digital and two conventional radiographic systems. Dentomaxillofac Radiol 2002; 31: 44-49.

2. Jacobsen JH, Hansen B, Wenzel A, Hintze H. Relationship between histological and radiographic caries lesion depth measured in images from four digital radiography systems. Caries Res 2004; 38: 34-38.

3. Furkart AJ, Dove SB, McDavid WD, Nummikoski P, Matteson S. Direct digital radiography for the detection of periodontal bone lesions. Oral Surg Oral Med Oral Pathol 1992; 74: 652-660.

4. Nair MK, Ludlow JB, Tyndall DA, Platin E, Denton G. Periodontitis detection efficacy of film and digital images. Oral Surg Oral Med Oral Pathol Oral Radiol Endod 1998; 85: 608-612.

5. Versteeg CH, Sanderink GC, van der Stelt PF. Efficacy of digital intra-oral radiography in clinical dentistry. J Dent 1997; 25: 215-224.

6. Baker LC. Benefits of interoperability: a closer look at the estimates. Health Aff (Millwood) 2005; 19: 22-25.

7. Benn DK, Bidgood WD Jr, Pettigrew JC Jr. An imaging standard for dentistry. Extension of the radiology DICOM standard. Oral Surg Oral Med Oral Pathol 1993; 76: 262-265.

8. Gotfredsen E, Wenzel A. A non-Dicom based system for distributing and viewing digital images from multiple sources in dentistry. In: Lemke HU, Vannier MW, Inamura K, Farman AG (eds). Computed assisted radiology and surgery. Amsterdam: Excerpta Medica; 1999: pp 964-967. might be related to the two-wall periodontal defect that was created, so that the remaining lingual wall was thick and might have produced a blurred image. In a study conducted by Pepelassi and Diamanti-Kipioti ${ }^{13}$ there was no reference to the type of bone defect (one-, two- or three-wall periodontal defects). In addition, we used a precision digital calliper to measure the bone defects instead of the periodontal probe used in previous reports. ${ }^{12,13}$

In conclusion, the periodontal bone defect measurements using the Schick dedicated software CDR DICOM for Windows v.3.5 and non-dedicated software Vix Win 2000 v.1.2, Image Tool 3.00 and Adobe Photoshop 7.0 were similar, while there was a considerable underestimation of bone defect depth measured in radiographs compared to dry mandibles.

\section{Acknowledgments}

This study was supported by Grants from Fundação de Amparo à Pesquisa do Estado de São Paulo \#02/13326-7 and \#02/13327-3.

\section{Disclosure}

The authors claim to have no financial interests in any company or any of the products mentioned in this manuscript.

9. Wenzel A, Møystad A. Experience of Norwegian general dental practitioners with solid state and storage phosphor detectors. Dentomaxillofac Radiol 2001; 30: 203-208.

10. Berkhout WE, Sanderink GC, van der Stelt PF. A comparison of digital and film radiography in Dutch dental practices assessed by questionnaire. Dentomaxillofac Radiol 2002; 31: 93-99.

11. Benediktsdottir IS. Digital panoramic radiography for assessment of mandibular third molars. [PhD thesis]. University of Aarhus, 2003.

12. Suomi JD, Plumbo J, Barbano JP. A comparative study of radiographs and pocket measurements in periodontal disease evaluation. J Periodontol 1968; 39: 311-315.

13. Pepelassi EA, Diamanti-Kipioti A. Selection of the most accurate method of conventional radiography for the assessment of periodontal osseous destruction. J Clin Periodontol 1997; 24: 557-567.

14. Akesson L, Hakansson J, Rohlin M. Comparison of panoramic and intraoral radiography and pocket probing for the measurement of the marginal bone level. J Clin Periodontol 1992; 19: 326-332. 